\title{
A Positioning Scheme Using Inertial Sensing for Indoor and Outdoor Environments
}

\author{
Yih-Shyh Chiou* and Fuan Tsai ${ }^{1}$ \\ Department of Electrical Engineering, Chinese Culture University, \\ No. 55, Hwakang Rd., Taipei 11114, Taiwan \\ ${ }^{1}$ Center for Space and Remote Sensing Research, National Central University, \\ No. 300, Jhongda Rd., Taoyuan 32001, Taiwan
}

(Received July 2, 2014; accepted May 11, 2015)

Key words: dead reckoning, error propagation, inertial measurement unit, location-based service, positioning system

Robust and accurate positioning techniques with seamless integration between indoor and outdoor environments have been receiving considerable attention for applications of location-based services (LBSs). Regarding positioning systems based on wireless network services, the location inaccuracy is caused by radio signals being jammed and/or shielded in outdoor environments and reflected and/or attenuated in indoor environments. As wireless positioning systems do not provide customers with adequate accuracy, inertial sensing positioning techniques can be used to deal with the inaccuracy problems for LBS applications. Consequently, in this study, we obtain displacement information by the relative positioning techniques according to the dead-reckoning (DR) approach to estimate the location of a mobile device (MD) for indoor and outdoor environments. Furthermore, instead of an MD mounted on a fixed place on a human body, the inertial measurement unit (IMU) is taken as a handheld MD (such as a smartphone) for positioning systems. In terms of the approach combining the DR algorithm with the heading and delta-velocity information extracted from an IMU sensor, the experimental results demonstrate that the positioning technique can recover and operate in a short period of time as the radio-based approaches cannot provide tailored accuracy for realtime LBS applications.

\section{Introduction}

Recently, location-based services (LBSs) have received considerable attention in many civilian and military applications. ${ }^{(1-4)}$ Hence, it is a fundamental problem to provide users with adequate location information for LBS applications. However, constructing a reliable and accurate positioning system to ensure continuous integration between indoor and outdoor environments is a great challenge..$^{(1-3)}$ In terms of wireless positioning systems, radio signals transmitted from base stations or navigation beacons ${ }^{*}$ Corresponding author: e-mail: choice@alumni.ncu.edu.tw 
transmitted from satellites are jammed or shielded in outdoor environments, and radio signals with the multipath effect are mostly reflected and attenuated by walls and furniture in indoor environments. ${ }^{(3)}$ As a result, many people in academia and industry are currently involved in the research and development of positioning techniques. In general, on the basis of the requirement of the location accuracy for LBS applications under different circumstances, there are two mechanisms applied in positioning systems. One is the absolute scheme (radio ranging) based on wireless network services, ${ }^{(1-3)}$ the other is the relative scheme (speed sensing) based on an inertial measurement unit (IMU). ${ }^{(5-8)}$ According to wireless network services, most LBSs use only the absolute scheme to determine the location of a mobile device (MD). In terms of global positioning system (GPS) approaches, they perform less well within forested areas, under poor geometric dilution of precision (GDOP) environments, and so forth. ${ }^{(9)}$ In terms of wireless fidelity (WiFi) approaches, because access points (APs) are rarely deployed to provide optimal geometry location, an MD cannot always receive radio signals transmitted from over three APs. ${ }^{(3)}$ Therefore, they result in sub-optimal positioning accuracy. Namely, estimating an accurate location using only an absolute scheme relying on a radio beacon or signal information remains a difficult problem concerning location accuracy. ${ }^{(3)}$

In recent years, IMU technologies have been developed for location-estimation and navigation systems. ${ }^{(5-8)}$ For relative positioning schemes, an MD based on lowcost micro-electromechanical systems (MEMS) sensors has made dead-reckoning (DR) algorithms attractive for LBS applications. When wireless positioning systems are not available, foot-mounted techniques based on DR approaches were used to calculate the moving distance of MDs. ${ }^{(5-8)}$ In addition, to the best of our knowledge, researchers seldom focus on similar approaches to continue and cover indoor and outdoor environments. Regarding the IMU sensing techniques in indoor environments, the foot-mounted or waist-mounted techniques were the most popular approaches. ${ }^{(5-8)}$ However, the main disadvantage of the foot-mounted configuration is that the approaches place complete reliance on an a priori step prediction algorithm. ${ }^{(6)}$ Consequently, understanding the features of the positioning approaches based on speed and attitude information is a research-worthy problem.

In this article, we introduce an IMU positioning approach based on the relative scheme to estimate the location of an MD (or car) for indoor and outdoor environments. Instead of the foot-mounted or waist-mounted approaches, the state-motion equations through calculation with the displacement formula are based on the heading and deltavelocity information, and then the positioning error caused by human factors can be reduced. According to the experimental results, without any absolute-scheme assistant, the IMU-based approach can estimate the locations of MDs in a short period of time in indoor and outdoor environments for LBS applications.

\section{System Description}

For positioning or navigation systems, a DR algorithm is one of the relative positioning schemes for calculating the current location according to previously 
determined locations and sensing information. ${ }^{(5-8)}$ According to the DR approach, the next location of an MD (or car) can be obtained from the calculated displacement in terms of speed observations and the estimated direction in terms of heading observations. ${ }^{(10,11)}$ The DR approach relies on initial information, it fuses heading and displacement information, and then the next location is estimated for positioning systems. In this article, the coordinate and attitude parameters are defined in Fig. 1; the location-estimation scheme described with the DR approach is illustrated in ref. 11. Regarding the relative positioning approach (2-D coordinate system), the mathematical models of the observation and of the system state at time $k$ can be taken as

Observation equation

$$
\theta_{k} \equiv f u n_{\theta}\left(x_{k}, y_{k}\right), V_{k} \equiv f u n_{\mathrm{S}}\left(a_{x, k}, a_{y, k}\right)
$$

State equation

$$
x_{k}=x_{0}+\sum_{i=0}^{k-1} d_{i} \cdot \cos \theta_{i}, k \geq 0, y_{k}=y_{0}+\sum_{i=0}^{k-1} d_{i} \cdot \sin \theta_{i}, k \geq 0,
$$

where $\operatorname{fun}_{\theta}(\cdot)$ and $\operatorname{fun}_{\mathrm{S}}(\cdot)$ are the heading transition function and speed transition function, respectively; $x_{0}$ and $y_{0}$ are the two coordinate values of the initial location at time $0 ; x_{k}$ and $y_{k}$ are the two coordinate values of the estimated location at time $k ; d_{i}$ and $\theta_{i}$ are the moving distance and observational absolute angle in terms of east at time $i$, respectively. As the main phases illustrated in Fig. 2, the DR approach is based on measurements of the current heading and delta velocity, and then the measurements are used to determine the changes from the initial position of the MD, which is named as the IMU-based

Define the parameters:

- Coordinate Direction $(X, Y, Z)$

- Attitude Orientation (Heading, Roll, Pitch)
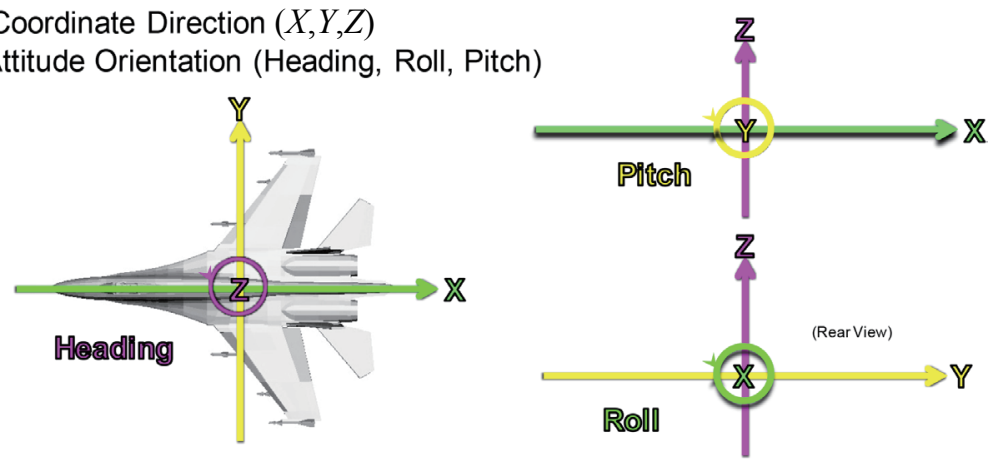

Fig. 1. (Color online) Definitions of the coordinate and attitude parameters. 


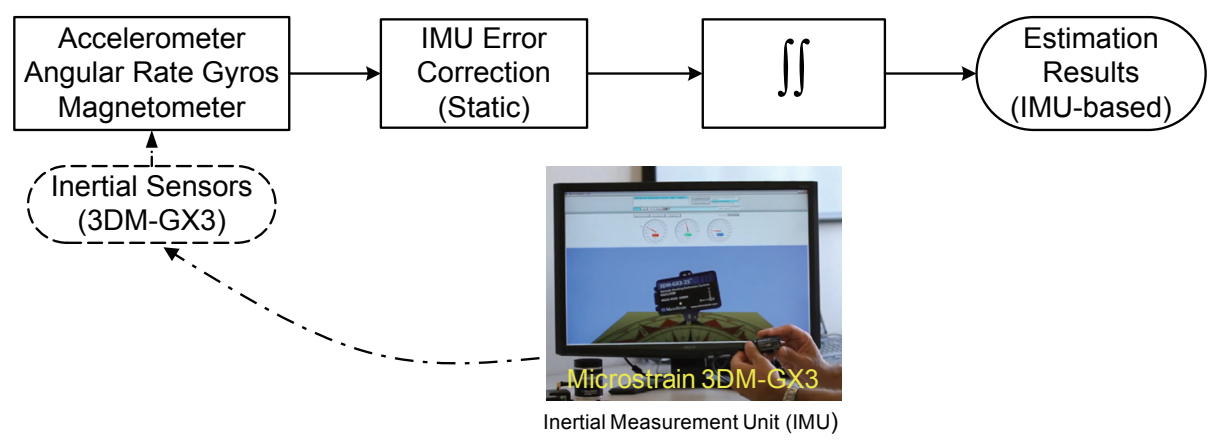

Fig. 2. (Color online) Location-estimation approach based on the delta-velocity information and attitude information.

approach. The models describing the motion of the MD given to verify the performance of the experiments are as follows: ${ }^{(11)}$

$$
\begin{aligned}
& x_{k+1}=x_{k}+\iint_{t_{k} \rightarrow t_{k+1}}\left(a_{x, k} \cos \theta_{k}-a_{y, k} \sin \theta_{k}\right) d \tau d t \\
& y_{k+1}=y_{k}+\iint_{t_{k} \rightarrow t_{k+1}}\left(a_{x, k} \sin \theta_{k}+a_{y, k} \cos \theta_{k}\right) d \tau d t,
\end{aligned}
$$

where the observations $a$ and $\theta_{k}$ are the acceleration and absolute angle, respectively, and then the acceleration information is integrated twice to yield the position. $\Delta_{k}$ is the measurement interval between the time $k\left(t_{k}\right)$ and the time $k+1\left(t_{k+1}\right)$.

\section{Experimental Results}

The objective of this study is to investigate the inertial sensing positioning approach based on the same mathematical models to continue and cover outdoor and indoor environments. As shown in Fig. 2, the IMU-based approach is based on attitude information and delta-velocity information supplied by the 3DM-GX3 IMU.(12) Not only does the IMU combine a triaxial accelerometer, gyro, and magnetometer, but it also provides the adjustable data rate of $1-1000 \mathrm{~Hz}$, and the $\mathrm{A} / \mathrm{D}$ resolution is 16 bits. In this study, the data rate of the IMU was set to 10,50 , or $100 \mathrm{~Hz}$ for different experiments. The heading information is defined as the angle of the MD in reference to true north, and the unit of heading information is degree(s). It is the moving direction of the MD, and the sensing values are in terms of north in the clockwise direction.

For the experiments located within outdoor environments, the 3DM-GX3 IMU was positioned flat (simulated as a handheld MD) in a car, and then the car was driven along specific experimental paths. To improve the location accuracy of the experimental results, the measurements of the first $60 \mathrm{~s}$ are used for static calibration. Therefore, the information sensing from the IMU is under the assumption that all the experiments have 
the same bias. The first experiment was carried out over a highway and a general road, and the car was driven along the environment illustrated in the left side of Fig. 3. As an MD moved along this specific path, the experimental results of the heading and the deltavelocity information are extracted from the IMU and illustrated in Fig. 4, and then the information is used to check the characteristics of the inertial sensor and to verify the performances of an IMU-based approach. The second experiment was carried out over a country road, and the car was driven along a general road of the suburbs around the city illustrated in the left side of Fig. 5. The third experiment was carried out within a campus, and the car was driven along a block area of a campus road illustrated in the left side of Fig. 6. In the case of the indoor environment, the fourth experiment was carried

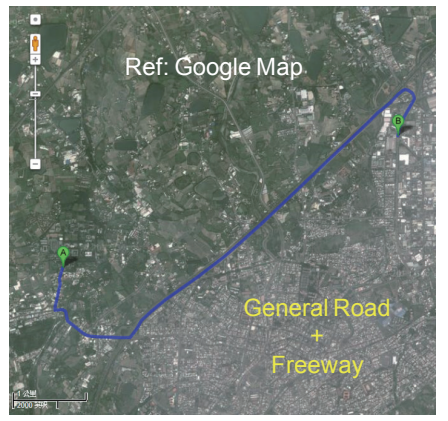

NCU $\rightarrow$ Jungyang Rd. $\rightarrow$ Minzu Rd

(a)

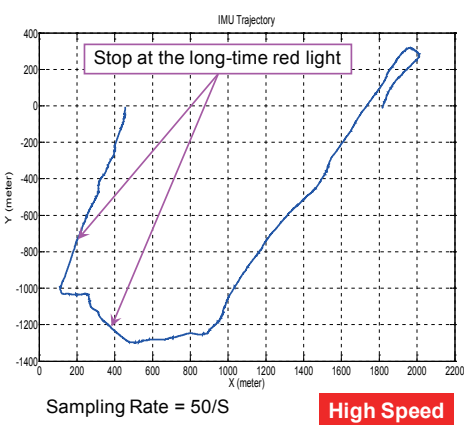

ungli Interchange $\rightarrow$ Neili Interchange

(b)

Fig. 3. (Color online) (a) Layout of the outdoor experimental environment (path: from A to B). (b) Location-estimation result of highway (freeway) and general road (high and intermediate speeds).
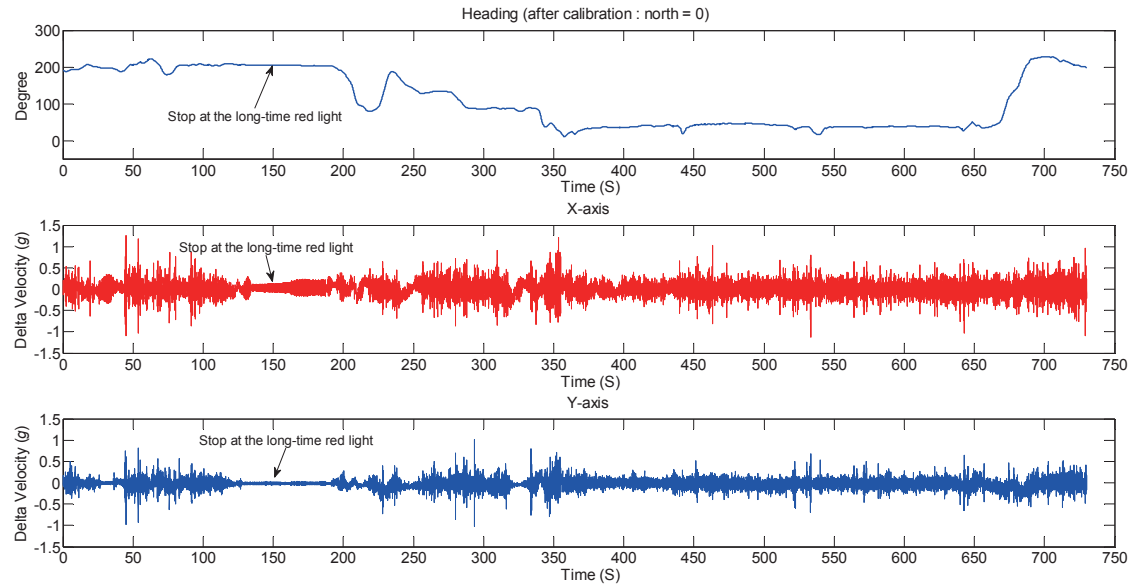

Fig. 4. (Color online) Measurements of heading and delta velocity in terms of data rate of the IMU set to 50 per second as an MD moved along a specific path in Fig. 3. 


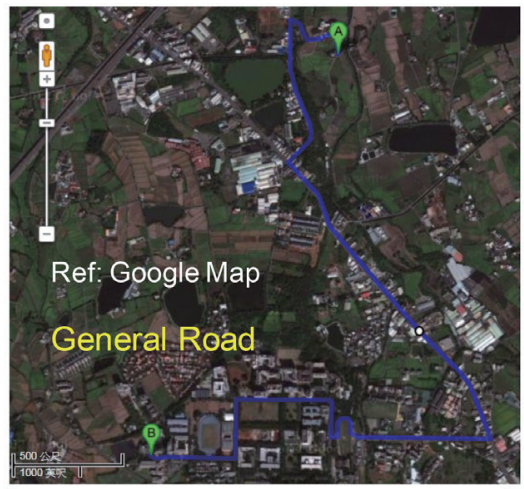

Shengde Rd. $\rightarrow$ Jhongcheng Rd. $\rightarrow$ Jhongda Rd. $\rightarrow$ NCU

(a)

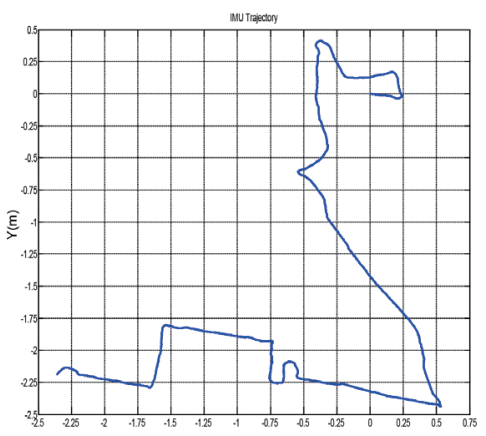

$\begin{array}{ll}\text { (2) Middle Speed } & x(m) \\ \text { Scale of Unit: } 1 / 500 \\ \text { Sampling Rate }=50 / 5\end{array}$

(b)

Fig. 5. (Color online) (a) Layout of the outdoor experimental environment (path: from A to B). (b) Location-estimation result of the country road (intermediate speed).

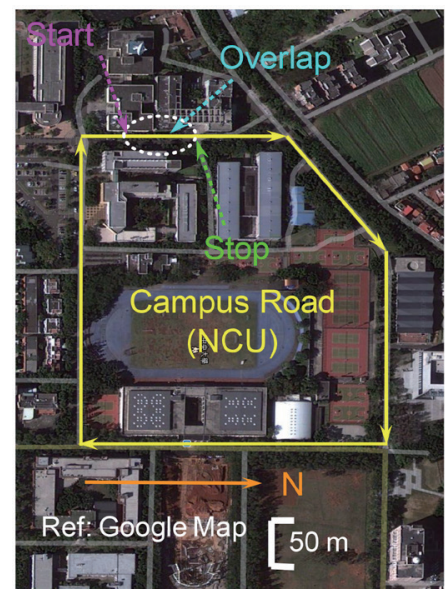

(a)

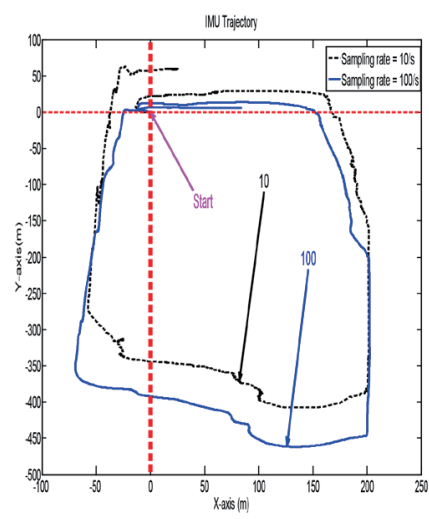

(3) Low Speed
Scale of Unit:1/1

(b)

Fig. 6. (Color online) (a) Layout of the outdoor experimental environment, National Central University (NCU) campus. (b) Location-estimation results of campus road (low speed).

out within a building, and a bird's-eye view of the hallway (in the building) is illustrated in the left side of Fig. 7.

In this study, the data rates of the IMU were set to 50 per second for the first and second experiments, 10 and 100 per second for the third experiment, and 100 per second for the fourth experiment. The experimental results shown in Figs. 3-6 revealed that the IMU-based approach could obtain the contours of the estimated MD's path, which is similar to the contours of a Google map in different environments. The experimental 


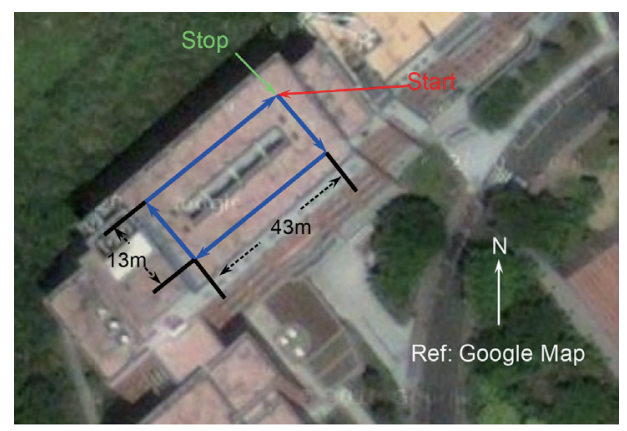

(a)

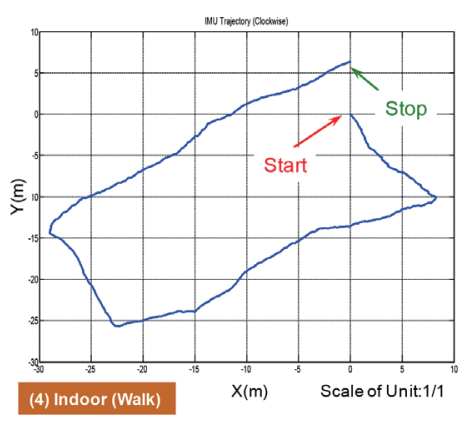

(b)

Fig. 7. (Color online) (a) Layout of the indoor experimental environment (a bird's-eye view of a building). (b) Location-estimation results as the MD moves in a clockwise direction of the hallway in the building.

results with different sampling rates shown in Fig. 6 revealed that the data rate of the IMU set to $100 \mathrm{~Hz}$ could predict the MD's trajectory, which is close to the true trajectory. Furthermore, the experimental results shown in Fig. 7 illustrate that the DR approach could obtain the contours of the estimated MD's path, which is similar to the contours of a hallway in an indoor environment. Nevertheless, in terms of the experimental results shown in Figs. 3-7 obtained by the IMU sensing approach, when an IMU was continually adding the detected changes to the current position, the observational errors accumulated. As illustrated in Fig. 2, the delta-velocity data are integrated twice to yield positions, and then the drift errors were enlarged. As a result, the accumulations of location errors became inevitable and unbounded with sensor biases and noises. ${ }^{(6,11)}$ Furthermore, as shown in Figs. 3 and 4, when the car was stopped for a prolonged red light, the vibration of the car (engine) caused serious location-estimation errors with the assumption that the same mean bias is through the same experimental environment. In addition, although the orientation errors of the IMU-based approach do not accumulate, the heading angle is extracted from an electric compass, and the measurement of heading angles is easily affected by the large distortions of the Earth's magnetic field near power lines or steel structures in different environments. Namely, the IMU-based approach using distance information and direction information for location estimation caused the error-propagation phenomenon for position or navigation applications. Regarding the error-propagation phenomenon, the character-recognition approach based on an event data recorder (EDR) information can be used to extract the names of streets as a landmark, and then the well-known location of a street can be used to calibrate and modify the estimated location to minimize the error-propagation phenomenon. ${ }^{(11)}$ In terms of the experiment results based on the DR approach, although the MD cannot properly track the path, the IMU-based approach is simple, inexpensive, and easy for accomplishing real-time positioning applications. Consequently, the IMU-based approach can considerably reduce the installation costs of positioning systems for LBS applications. 


\section{Conclusions}

In this article, we have examined the positioning algorithm obtaining displacement information through calculation with the observed angle and speed information according to the relative positioning approach and the displacement formula. For reasonable factors of the experiments, the IMU sensor is taken as a handheld MD (such as smartphone), and it is not mounted on a fixed place on a human body. The experimental results revealed that the positioning errors of the IMU-based approach grow with time, and the paths of MDs cannot be properly estimated in terms of IMU observations. However, the IMUbased approach can still position the MD's location accurately in a short period of time, and then the IMU-based positioning system allows users to know their positions and to obtain the contours of MDs. Consequently, regarding seamless indoor and outdoor environments with the same handheld MD for real-time LBS applications, as the radio signals based on wireless network services cannot be used to estimate the locations of MDs, the approach based on attitude information and delta-velocity information extracted from an IMU can be used to improve the location estimation of the MD of a positioning system.

\section{Acknowledgements}

This work was supported by the Ministry of Science and Technology (National Science Council) of the Republic of China under Grants NSC 101-2218-E-033-007, NSC 102-2221-E-033-032 (MOST 102-2221-E-034-021), and MOST 103-2221-E-034-020.

\section{References}

1 Y.-S. Chiou and F. Tsai: IEEE Trans. Cybern. 44 (2014) 922.

2 J. Rantakokko, J. Rydell, P. Stromback, P. Handel, J. Callmer, D. Tornqvist, F. Gustafsson, M. Jobs, M. Gruden and M. Gezici: IEEE Wireless Commun. 18 (2011) 10.

3 R. J. Barton, R. Zheng, S. Gezici and V. V. Veeravalli: EURASIP J. Adv. Sig. Process. 2008 (2008) 1 .

4 Y.-L. Chen and X.-Z. Shi: IJETI 2 (2012) 216.

5 H. Wang, H. Lenz, A. Szabo, J. Bamberger and U. D. Hanebeck: Proc. WPNC '07 (IEEE, 2007) pp. 1-7.

6 J. Bird and D. Arden: IEEE Wireless Commun. 18 (2011) 28.

7 R. Zhang, F. Höflinger and L. Reindl: IEEE Sens. J. 13 (2013) 838.

8 H. Fourati: IEEE Trans. Instrum. Meas. 64 (2015) 221.

9 E. D. Kaplan and C. J. Hegarty: Understanding GPS: Principles and Applications (Artech House, Massachusetts, 2006) pp. 322-328, 529.

10 Y.-S. Chiou and F. Tsai: Proc. ICETI2014 (ICETI, 2014) pp. 550-554.

11 Y.-S. Chiou, F. Tsai and S.-C. Yeh: Proc. IIH-MSP-2014 (IEEE, 2014) pp. 377-380.

12 Microstrain Inc.: http://www.microstrain.com/inertial. 